PROCEEDINGS OF THE

AMERICAN MATHEMATICAL SOCIETY

Volume 136, Number 8, August 2008, Pages 2745-2747

S 0002-9939(08)09470-7

Article electronically published on April 3, 2008

\title{
TRIANGULATED CATEGORIES OF GORENSTEIN CYCLIC QUOTIENT SINGULARITIES
}

\author{
KAZUSHI UEDA \\ (Communicated by Ted Chinburg)
}

\begin{abstract}
We prove there is an equivalence of derived categories between Orlov's triangulated category of singularities for a Gorenstein cyclic quotient singularity and the derived category of representations of a quiver with relations, which is obtained from a McKay quiver by removing one vertex and half of the arrows. This result produces examples of distinct quivers with relations which have equivalent derived categories of representations.
\end{abstract}

Fix an integer $n$ greater than one. For a finite subgroup $G$ of $G L_{n}(\mathbb{C})$, let $\left\{\rho_{i}\right\}_{i=0}^{N-1}$ be the set of irreducible representations of $G$. Further, let $\rho_{\text {Nat }}$ be the natural $n$-dimensional representation of $G$ given by the inclusion. For $k, l=0, \ldots, N-1$, define the natural numbers $a_{k l}$ by the decomposition

$$
\rho_{l} \otimes \rho_{\mathrm{Nat}}=\bigoplus_{k} \rho_{k}^{\oplus a_{k l}}
$$

of the tensor product into the direct sum of irreducible representations. The McKay quiver of $G$ is the quiver whose set of vertices is $\left\{\rho_{i}\right\}_{i=0}^{N-1}$ and the number of whose arrows from the vertex $\rho_{k}$ to the vertex $\rho_{l}$ is $a_{k l}$ [5].

Now assume that $G$ is a cyclic group generated by an element of the form $g=$ $\operatorname{diag}\left(\zeta^{a_{1}}, \ldots, \zeta^{a_{n}}\right)$, where $a_{1}, \ldots, a_{n}$ are positive integers such that $\operatorname{gcd}\left(a_{1}, \ldots, a_{n}\right)$ $=1$ and $\zeta=\exp \left[2 \pi \sqrt{-1} /\left(a_{1}+\cdots+a_{n}\right)\right]$. Let $R=\mathbb{C}\left[x_{1}, \ldots, x_{n}\right]$ be the polynomial ring in $n$ variables equipped with the $\mathbb{Z}$-grading given by $\operatorname{deg} x_{i}=a_{i}, i=1, \ldots, n$. Define another $\mathbb{Z}$-graded ring $A\left(a_{1}, \ldots, a_{n}\right)=\bigoplus_{k \geq 0} A_{k}$ by

$$
A_{k}=R_{k\left(a_{1}+\cdots+a_{n}\right)} \text {. }
$$

Then $A\left(a_{1}, \ldots, a_{n}\right)$ is the invariant ring of $R$ by the action of $G$ so that $\mathbb{C}^{n} / G=$ $\operatorname{Spec} A\left(a_{1}, \ldots, a_{n}\right)$.

In this case, the corresponding McKay quiver has $N=a_{1}+\cdots+a_{n}$ vertices $\left\{\rho_{k}\right\}_{k=0}^{N-1}$ and $n N$ arrows $\left\{x_{i, k}\right\}_{i=1, \ldots, n}, \begin{aligned} k \\ k=0, \ldots, N-1\end{aligned}$, where $\rho_{k}$ is the one-dimensional representation of $G$ sending $g \in G$ to $\exp \left[-2 k \pi \sqrt{-1} /\left(a_{1}+\cdots+a_{n}\right)\right] \in \mathbb{C}^{\times}$, and $x_{i, k}$ is an arrow from $\rho_{k}$ to $\rho_{k+a_{i}}$.

Next we introduce another quiver $Q\left(a_{1}, \ldots, a_{n}\right)$ obtained by removing the vertex $\rho_{0}$ and half of the arrows from the McKay quiver. The set of vertices of

Received by the editors July 6, 2006, and, in revised form, October 6, 2006, and July 8, 2007. 2000 Mathematics Subject Classification. Primary 18E30; Secondary 16G20.

The author was supported by the 21st Century COE Program of Osaka University. 
$Q\left(a_{1}, \ldots, a_{n}\right)$ is $\left\{\rho_{k}\right\}_{k=1}^{N}$, and an arrow of the McKay quiver from $\rho_{k}$ to $\rho_{l}$ is an arrow of $Q_{g}$ if $0<k<l$.

A quiver with relations is a pair $\Gamma=(Q, \mathcal{I})$ of a quiver $Q$ and a two-sided ideal $\mathcal{I}$ of its path algebra $\mathbb{C} Q$. We equip $Q\left(a_{1}, \ldots, a_{n}\right)$ with the relations $\mathcal{I}\left(a_{1}, \ldots, a_{n}\right)$ generated by $x_{j, k+a_{i}} x_{i, k}-x_{i, k+a_{j}} x_{j, k}$ for $1 \leq i<j \leq n$ and $k=1, \ldots, N-a_{i}-a_{j}-1$, and put $\Gamma\left(a_{1}, \ldots, a_{n}\right)=\left(Q\left(a_{1}, \ldots, a_{n}\right), \mathcal{I}\left(a_{1}, \ldots, a_{n}\right)\right)$. The main theorem is:

Theorem 1. For a sequence $a_{1}, \ldots, a_{n}$ of positive integers such that $\operatorname{gcd}\left(a_{1}, \ldots, a_{n}\right)$ $=1$, there exists an equivalence

$$
D_{\mathrm{Sg}}^{\mathrm{gr}}\left(A\left(a_{1}, \ldots, a_{n}\right)\right) \cong D^{b} \bmod \Gamma\left(a_{1}, \ldots, a_{n}\right)
$$

of triangulated categories.

Here, $D^{b} \bmod \Gamma\left(a_{1}, \ldots, a_{n}\right)$ is the bounded derived category of finite-dimensional right modules over the path algebra $\mathbb{C} \Gamma\left(a_{1}, \ldots, a_{n}\right)=\mathbb{C} Q\left(a_{1}, \ldots, a_{n}\right) / \mathcal{I}\left(a_{1}, \ldots, a_{n}\right)$ with relations. The above theorem produces examples of distinct quivers with relations with equivalent derived categories of representations; e.g. when $n=3$, the $\mathbb{Z}$-graded rings $A(1,2,2)$ and $A(3,1,1)$ are isomorphic, although the quivers with relations $\Gamma(1,2,2)$ and $\Gamma(3,1,1)$ are not isomorphic.

$D_{\mathrm{Sg}}^{\mathrm{gr}}\left(A\left(a_{1}, \ldots, a_{n}\right)\right)$ is the triangulated category of singularities defined by Orlov [6] as the quotient category

$$
D_{\mathrm{Sg}}^{\mathrm{gr}}\left(A\left(a_{1}, \ldots, a_{n}\right)\right)=D^{b} \operatorname{gr} A\left(a_{1}, \ldots, a_{n}\right) / D^{b} \operatorname{grproj} A\left(a_{1}, \ldots, a_{n}\right)
$$

of the bounded derived category $D^{b}$ gr $A\left(a_{1}, \ldots, a_{n}\right)$ of finitely-generated $\mathbb{Z}$-graded $A\left(a_{1}, \ldots, a_{n}\right)$-modules by its full triangulated subcategory $D^{b}$ grproj $A\left(a_{1}, \ldots, a_{n}\right)$ consisting of perfect complexes. The $n=2$ case in the above theorem is due to Takahashi [7] (see also Kajiura, Saito, and Takahashi [4).

The proof goes as follows: Let

$$
\text { qgr } R:=\operatorname{gr} R / \text { tor } R
$$

be the quotient category of the abelian category gr $R$ of finitely-generated $\mathbb{Z}$-graded $R$-modules by its full subcategory tor $R$ consisting of torsion modules, and let $\pi$ : $\operatorname{gr} R \rightarrow \operatorname{qgr} R$ be the natural projection functor. For $M \in \operatorname{gr} R$ and $l \in \mathbb{Z}, M(l)$ denotes the graded $R$-module shifted by $l ; M(l)_{k}=M_{l+k}$. Define a shift operator $s:$ qgr $R \rightarrow$ qgr $R$ by $s(\pi M)=\pi M\left(a_{1}+\cdots+a_{n}\right)$ and put $\mathcal{O}=\pi R$. Then one has $A\left(a_{1}, \ldots, a_{n}\right)=\bigoplus_{k=0}^{\infty} \operatorname{Hom}\left(\mathcal{O}, s^{k}(\mathcal{O})\right)$. Since $\operatorname{gcd}\left(a_{1}, \ldots, a_{n}\right)=1$, the graded $R$-module $R(l)$ for any $l \in \mathbb{Z}$ is generated up to torsion modules by the subset $\bigcup_{j \in \mathbb{N}} R(l)_{j\left(a_{1}+\cdots+a_{n}\right)}$ consisting of elements whose degrees are positive multiples of $a_{1}+\cdots+a_{n}$. Hence $s$ is ample and one has

$$
\operatorname{qgr} R \cong \operatorname{qgr} A\left(a_{1}, \ldots, a_{n}\right)
$$

by Artin and Zhang [1, Theorem 4.5].

Since $s^{-1}(\mathcal{O})$ is the dualizing sheaf, $A\left(a_{1}, \ldots, a_{n}\right)$ is Gorenstein with Gorenstein parameter 1 (cf. 6, Lemma 2.11]). Therefore one has a semiorthogonal decomposition

$$
D^{b} \operatorname{qgr} A\left(a_{1}, \ldots, a_{n}\right)=\left\langle\mathcal{O}, D_{\mathrm{Sg}}^{\mathrm{gr}}\left(A\left(a_{1}, \ldots, a_{n}\right)\right)\right\rangle
$$

by Orlov [6, Theorem 2.5(i)]. Here, $D^{b} \operatorname{qgr} A\left(a_{1}, \ldots, a_{n}\right)$ denotes the bounded derived category of the abelian category $\operatorname{qgr} A\left(a_{1}, \ldots, a_{n}\right)$. On the other hand, $D^{b}($ qgr $R)$ has a full strong exceptional collection $\left(\mathcal{O}, \mathcal{O}(1), \ldots, \mathcal{O}\left(a_{1}+a_{2}+\cdots+\right.\right.$ $\left.\left.a_{n}-1\right)\right)$ (see e.g. [2, Theorem 2.12]). Hence $D_{\mathrm{Sg}}^{\mathrm{gr}}\left(A\left(a_{1}, \ldots, a_{n}\right)\right)$ is equivalent to the 
full triangulated subcategory of $D^{b}$ qgr $A\left(a_{1}, \ldots, a_{n}\right)$ generated by the exceptional collection $\left(\mathcal{O}(1), \ldots, \mathcal{O}\left(a_{1}+\cdots+a_{n}-1\right)\right)$. By Bondal [3, Theorem 6.2], this subcategory is isomorphic to the derived category of finite-dimensional right modules over the total morphism algebra

$$
\bigoplus_{i, j=1}^{a_{1}+\cdots+a_{n}-1} \operatorname{Hom}(\mathcal{O}(i), \mathcal{O}(j))
$$

of this collection, which is isomorphic to $\mathbb{C} \Gamma\left(a_{1}, \ldots, a_{n}\right)$.

\section{REFERENCES}

[1] M. Artin and J. J. Zhang. Noncommutative projective schemes. Adv. Math., 109(2):228-287, 1994. MR1304753 (96a:14004)

[2] Denis Auroux, Ludmil Katzarkov, and Dmitri Orlov. Mirror symmetry for weighted projective planes and their noncommutative deformations. math.AG/0404281, 2004.

[3] A. I. Bondal. Representations of associative algebras and coherent sheaves. Izv. Akad. Nauk SSSR Ser. Mat., 53(1):25-44, 1989. MR992977 (90i:14017)

[4] Hiroshige Kajiura, Kyoji Saito, and Atsushi Takahashi. Matrix factorizations and representations of quivers II: type ADE case. math.AG/0511155, 2005.

[5] John McKay. Graphs, singularities, and finite groups. In The Santa Cruz Conference on Finite Groups (Univ. California, Santa Cruz, Calif., 1979), volume 37 of Proc. Sympos. Pure Math., pages 183-186. Amer. Math. Soc., Providence, R.I., 1980. MR604577 (82e:20014)

[6] D. O. Orlov. Derived categories of coherent sheaves and triangulated categories of singularities. math.AG/0503632, 2005.

[7] Atsushi Takahashi. Matrix factorizations and representations of quivers I. math.AG/0506347, 2005.

Department of Mathematics, Graduate School of Science, Osaka University, Machikaneyama 1-1, TOYONAKa, OSAKa, 560-0043, Japan

E-mail address: kazushi@math.sci.osaka-u.ac.jp 